J. Korean Math. Soc. 48 (2011), No. 3, pp. 475-486

DOI 10.4134/JKMS.2011.48.3.475

\title{
REPEATED LOW-DENSITY BURST ERROR DETECTING CODES
}

\author{
Bal Kishan Dass and Rashmi Verma
}

\begin{abstract}
The paper deals with repeated low-density burst error detecting codes with a specified weight or less. Linear codes capable of detecting such errors have been studied. Further codes capable of correcting and simultaneously detecting such errors have also been dealt with. The paper obtains lower and upper bounds on the number of parity-check digits required for such codes. An example of such a code has also been provided.
\end{abstract}

\section{Introduction}

One of the most important aspects in Coding Theory has been the detection and correction of errors. Various kinds of errors have been dealt with for which codes have been developed to detect and/or correct such errors. Hamming [8] made the beginning with the detection and correction of random errors. Considering the fact that in many communication channels the likelihood of the occurrence of errors is more in adjacent digits rather than their occurrence in a random manner, Abramson [1] developed codes which dealt with the correction of single and double adjacent errors. A more general concept of clustered errors called 'burst errors' was given by Fire [7]. A burst of length $b$ is defined as follows:

Definition 1. A burst of length $b$ is a vector whose only non-zero components are among some $b$ consecutive components, the first and the last of which is non-zero.

Fire [7] considered two kinds of bursts viz., 'open-loop burst' which are popularly refered to simply a burst as in Definition 1 and the other called 'closed-loop burst' defined as follows:

Definition 2. Let $b$ be an integer and $x=\left(\xi_{1}, \xi_{2}, \ldots, \xi_{n}\right)$ be a vector in $V^{n}(q)$, a vector space of $n$-tuples over $\operatorname{GF}(q)$. If $2 \leq b \leq(n+1) / 2$, then $x$ is

Received November 18, 2009

2010 Mathematics Subject Classification. 94B20, 94B25, 94B65.

Key words and phrases. error detecting code, burst error, low-density burst error, repeated low-density burst error. 
called a 'closed-loop burst vector of length $b$ ' whenever there is an $i$ such that $1 \leq i \leq b-1$,

$$
\xi_{i} \cdot \xi_{n-b+i+1} \neq 0 ; \quad \xi_{i+1}=\xi_{i+2}=\ldots=\xi_{n-b+i}=0 .
$$

Apart from random errors, one of the widely studied error is a burst error. Burst error correcting codes are needed in virtually unaccountable applications. Since the development of various burst error detecting and correcting codes, several variants and modifications of the burst error came up depending upon the various kinds of channels which were in use. The nature of burst errors differ from channel to channel depending upon the type of channels or the errors which occur during the process of transmission. In very busy communication channels, errors repeat themselves. Dass and Verma [5] studied repeated burst errors. They termed such a burst error as ' $m$-repeated burst of length $b$ ' which has been defined as follows:

Definition 3. An $m$-repeated burst of length $b$ is a vector of length $n$ whose only non-zero components are confined to $m$ distinct sets of $b$ consecutive components, the first and the last component of each set being non-zero.

During the process of transmission some disturbances cause occurrence of burst errors in such a way that over a given length, some digits are received correctly while others are corrupted, i.e., not all digits inside a burst are in error. A. D. Wyner [13] termed such bursts as low-density bursts defined as follows:

Definition 4. A low-density burst of length $b$ with weight $w$ is an $n$-tuple whose only non-zero components are confined to $b$ consecutive positions, the first and the last of which is non-zero, with $w(w \leq b)$ non-zero components within such $b$ consecutive digits.

Further study on low-density burst error detecting and correcting linear codes has been made by Sharma and Dass [12] and Dass [3, 4].

In this paper, we consider codes which are capable to detect/correct repeated low-density bursts of length $b$ or less with weight $w$ or less. An $m$-repeated lowdensity burst is defined as follows:

Definition 5. An $m$-repeated low-density burst of length $b$ with weight $w$ is a vector of length $n$ whose only non-zero components are confined to $m$ distinct sets of $b$ consecutive components, the first and the last component of each set being non-zero, with $w(w \leq b)$ non-zero components within each set of such $b$ consecutive components.

For example: (010120120400301200) is a 3-repeated low-density burst of length 4 with weight 3 over $\mathrm{GF}(5)$.

In particular, a 2-repeated low-density burst of length $b$ with weight $w(w \leq$ b) is defined as follows: 
Definition 6. A 2-repeated low-density burst of length $b$ with weight $w$ is a vector of length $n$ whose only non-zero components are confined to two distinct sets of $b$ consecutive components, the first and the last component of each set being non-zero, with $w(w \leq b)$ non-zero components within each set of such $b$ consecutive components.

For example, (010203002001400) is a 2-repeated low-density burst of length 5 with weight 3 over GF(5).

The development of codes detecting and correcting repeated low-density burst errors will economize in the number of parity-check digits in comparison to the usual low-density burst error detecting and correcting codes.

The paper has been organized as follows:

In Section 2, we derive lower and upper bound for codes detecting 2-repeated low-density burst errors of length $b$ or less with weight $w$ or less. Section 3 presents a bound for codes which can correct and simultaneously detect 2repeated low-density bursts of length $b$ or less with weight $w$ or less. In Section 4 , we obtain a lower bound for codes detecting $m$-repeated low-density bursts of length $b$ or less with weight $w$ or less followed by another bound for codes which can correct and simultaneously detect such repeated low-density bursts. The paper concludes with an illustration of a 2-repeated low-density burst of length 3 or less with weight 2 or less detecting code over GF(2).

In what follows a linear code will be considered as a subspace of the space of all $n$-tuples over $\operatorname{GF}(q)$. The distance between two vectors shall be considered in the Hamming sense.

\section{2-repeated low-density burst error detecting codes}

In this section, we consider linear codes that are capable of detecting any 2-repeated low-density burst of length $b$ or less with weight $w$ or less. Clearly, the patterns to be detected should not be code words. In other words, we consider linear codes that have no 2- repeated low-density burst of length $b$ or less with weight $w$ or less as a code word. Firstly, we obtain a lower bound on the number of parity-check digits required for such a code.

Theorem 1. An $(n, k)$ linear code over $\mathrm{GF}(q)$ that detects any 2-repeated lowdensity burst of length $b$ or less with weight $w$ or less must have at least $2 w$ parity-check digits $(w \leq b)$.

Proof. The result will be proved on the basis that no detectable error vector can be a code word.

Let $V$ be an $(n, k)$ linear code over $\operatorname{GF}(q)$. Consider the set $X$ that has all those vectors which have their non-zero components confined to the first $2 b$ components such that from each set of $b$ consecutive components, i.e., 1st to $b$-th and $(b+1)$-th to $2 b$-th components, the non-zero components are confined to some fixed $w(w \leq b)$ components. 
We claim that no two vectors of $X$ can belong to the same coset of the standard array, else a code word shall be expressible as a sum or difference of two error vectors.

Assume, on the contrary, that there is a pair say $x_{1}, x_{2}$ in $X$ belonging to the same coset of the standard array. Their difference viz., $x_{1}-x_{2}$ must be a code word. But $x_{1}-x_{2}$ is a vector all of whose non-zero components are confined to the first $2 b$ components with non-zero components confining to the same fixed $w$ or less non-zero components each in 1 st to $b$-th and $(b+1)$-th to $2 b$-th components and so is a member of $X$, i.e., $x_{1}-x_{2}$ is 2-repeated low-density burst of length $b$ or less with weight $w$ or less, which is a contradiction.

Thus all the vectors in $X$ must belong to distinct cosets of the standard array. The number of such vectors over $\operatorname{GF}(q)$ is clearly $q^{2 w}$. Also, total number of cosets in an $(n, k)$ linear code equals $q^{n-k}$, so we must have $q^{n-k} \geq q^{2 w}$, i.e., $n-k \geq 2 w$, which proves the result.

Remark 1. For $w=b$, the result reduces to Theorem 1 of Berardi, Dass, and Verma [2] when the bursts considered are 2-repeated bursts of length $b$ or less.

In the following result, we derive another bound on the number of check digits required for the existence of such a code. The proof is based on the technique used to establish Varsharmov-Gilbert-Sacks bound by constructing a parity-check matrix for such a code (refer Theorem 4.7, Peterson and Weldon $[9]$ ). This technique not only ensures the existence of such a code but also gives a method for the construction of such a code.

Theorem 2. There shall always exist an $(n, k)$ linear code over $\mathrm{GF}(q)$ that has no 2-repeated low-density burst of length $b$ or less with weight $w$ or less $(w \leq b)$ as a code word provided that

$$
\begin{aligned}
q^{n-k}> & {[1+(q-1)]^{(b-1, w-1)}\left\{q^{w-1}((q-1)(n-b-w+1)+1)+(q-1)^{2}\right.} \\
& \left.\sum_{i=w+1}^{b}(n-b-i+1)[1+(q-1)]^{(i-2, w-2)}\right\}+\sum_{i=w}^{2 w-1}\left(\begin{array}{c}
b-1 \\
i
\end{array}\right)(q-1)^{i} \\
& +\sum_{k=1}^{b-1} \sum_{r_{1}, r_{2}, r_{3}}\left(\begin{array}{c}
b-k-1 \\
r_{1}
\end{array}\right)\left(\begin{array}{c}
k \\
r_{2}
\end{array}\right)\left(\begin{array}{c}
b-k-1 \\
r_{3}
\end{array}\right)(q-1)^{r_{1}+r_{2}+r_{3}+1},
\end{aligned}
$$

where $0 \leq r_{1} \leq w-2,1 \leq r_{2} \leq 2 w-2,0 \leq r_{3} \leq w-1, r_{2}+r_{3} \geq w$, $r_{1}+r_{2}+r_{3} \leq 2 \bar{w}-2$, and $[1+x]^{(m, r)}$ denotes the incomplete binomial expansion of $(1+x)^{m}$ upto the term $x^{r}$ in ascending powers of $x$.

Proof. We shall prove the result by constructing an appropriate $(n-k) \times n$ parity-check matrix $H=\left[h_{1}, h_{2}, \ldots, h_{n}\right]$ for the desired code. Choose any non-zero $(n-k)$-tuple as the first column $h_{1}$ of $H$. Suppose that we have selected the first $(j-1)$ columns $h_{1}, h_{2}, \ldots, h_{j-1}$ of $H$ suitably. We lay down the condition to add the $j$ th column $h_{j}$ as follows: 
$h_{j}$ should not be a linear combination of any $w-1$ or fewer columns of the immediately preceding $(b-1)$ or fewer columns of $H$ together with any $w$ or less columns from any $b$ or fewer consecutive columns amongst the first $j-1$ columns. In other words,

(2) $h_{j} \neq\left(\alpha_{1} h_{i_{1}}+\alpha_{2} h_{i_{2}}+\cdots+\alpha_{w-1} h_{i_{w-1}}\right)+\left(\beta_{1} h_{j_{1}}+\beta_{2} h_{j_{2}}+\ldots+\beta_{w} h_{j_{w}}\right)$,

where $\alpha_{i}, \beta_{i} \in \operatorname{GF}(q)$ and the $h_{i}$ are any $(w-1)$ columns among $h_{j-b+1}, h_{j-b+2}$, $\ldots, h_{j-1}$ and the $h_{j_{1}}, h_{j_{2}}, \ldots, h_{j_{w}}$ are any $w$ columns from a set of $b$ consecutive columns amongst the first $(j-1)$ columns.

This condition ensures that no 2-repeated low-density burst of length $b$ or less with weight $w$ or less will be a code word.

The above inequality (2) is same as the condition laid down for the correction of single (usual) low-density burst of length $b$ or less with weight $w$ or less (refer B. K. Dass [4]) leading to the same computational result and is therefore being omitted.

Remark 2. In view of the fact that the result obtained in Theorem 2 is same as the result for the correction of low-density bursts of length $b$ or less with weight $w$ or less, such a code can serve a dual purpose, i.e., it can either be used to correct bursts of length $b$ or less with weight $w$ or less, or can be used to detect a 2-repeated low-density burst of length $b$ or less with weight $w$ or less.

\section{Simultaneous detection and correction of 2-repeated low-density burst errors}

In the following, we consider linear codes which are capable to correct and simultaneously detect 2-repeated low-density bursts with weight $w$ or less and obtain a necessary condition over the number of parity-checks required for such a code.

Theorem 3. Any $(n, k)$ linear code over $\operatorname{GF}(q)$ that corrects all 2-repeated lowdensity bursts of length $b$ or less with weight $w$ or less $(w \leq b)$ must have at least $4 w$ parity-check digits. Further, if the code corrects all 2-repeated low-density bursts of length $b$ or less with weight $w_{1}$ or less $\left(w_{1} \leq b\right)$ and simultaneously detects 2-repeated low-density bursts of length $d$ or less $(d \geq b)$ with weight $w_{2}$ or less $\left(w_{2} \leq d\right)$, then the code must have at least $2\left(w_{1}+w_{2}\right)$ parity-check digits.

Proof. Consider a vector all of whose non-zero components are confined to the first $4 b$ components such that from each set of $b$ consecutive components, i.e., 1 st to $b$-th, $(b+1)$-th to $2 b$-th, $(2 b+1)$-th to $3 b$-th and $(3 b+1)$-th to $4 b$-th components, the non-zero components are confined to some fixed $w(w \leq b)$ components. Such a vector is expressible as a sum or difference of two vectors each of which is a 2-repeated low-density burst of length $b$ or less with weight 
$w$ or less. These component vectors must belong to different cosets of the standard array because both such errors are correctable errors.

Accordingly, such a vector viz., low-density burst of length $4 b$ or less with weight $4 w$ or less cannot be a code word. In view of Theorem 1 , such a code must have at least $4 w$ parity-check digits.

Further, consider a vector whose only non-zero components are confined to the first $2(b+d)$ components. It may be noted that such a vector is expressible as a sum of four vectors where two of these are bursts of length $b$ or less each and the other two are bursts of length $d$ or less each. In each set of $b$ consecutive components, the non-zero components are confined to some fixed $w_{1}\left(w_{1} \leq b\right)$ components whereas in each set of $d$ consecutive components, the non-zero components are confined to some fixed $w_{2}\left(w_{2} \leq d\right)$ components. Obviously, such a vector is expressible as a sum or difference of two vectors, one of which is a 2-repeated low-density burst of length $b$ or less with weight $w_{1}$ or less and the other is a 2-repeated low-density burst of length $d$ or less with weight $w_{2}$ or less. Both such component vectors, one being a detectable error and the other being a correctable error, cannot belong to the same coset of the standard array. Therefore, such a vector cannot be a code word, i.e., a low-density burst of length $2(b+d)$ or less with weight $2\left(w_{1}+w_{2}\right)$ or less cannot be a code vector. In view of Theorem 1 , such a code must have at least $2\left(w_{1}+w_{2}\right)$ parity-check digits.

Remark 3. For $w=w_{1}=b$ and $w_{2}=d$, the result reduces to Theorem 3 of Berardi, Dass, and Verma [2] when the bursts considered are 2-repeated bursts of length $b$ or less.

\section{4. $m$-repeated low-density burst error detecting codes}

In this section, we consider linear codes that are capable to detect any $m$ repeated low-density burst of length $b$ or less with weight $w$ or less. Clearly, the patterns to be detected should not be code words. In other words, we consider linear codes that have no $m$-repeated low-density burst of length $b$ or less with weight $w$ or less as a code word. Firstly, we obtain a lower bound over the number of parity-check digits for such a code.

Theorem 4. An $(n, k)$ linear code over $\mathrm{GF}(q)$ that detects any m-repeated low-density burst of length $b$ or less with weight $w$ or less $(w \leq b)$ must have at least mw parity-check digits.

Proof. The result will be proved on the basis that no detectable error vector can be a code word.

Let $V$ be an $(n, k)$ linear code over $\operatorname{GF}(q)$. Consider a set $X$ that has all those vectors which have their non-zero components confined to the first $m b$ components such that from each set of $b$ consecutive components, i.e., $(i b+1)$ th to $(i+1) b$-th components, $i=0,1, \ldots,(m-1)$, the non-zero components are confined to some fixed $w(w \leq b)$ components. 
We claim that no two vectors of $X$ can belong to the same coset of the standard array, else a code word would be expressible as a sum or difference of two error vectors.

Assume, on the contrary, that there is a pair say $x_{1}, x_{2}$ in $X$ belonging to the same coset of the standard array. Then their difference viz., $x_{1}-x_{2}$ must be a code word. But $x_{1}-x_{2}$ is a vector all of whose non-zero components are confined to the first $m b$ components with non-zero components confining to the same fixed $w$ or less non-zero components each in the $(i b+1)$-th to $(i+1) b$-th components, $i=0,1, \ldots(m-1)$ and so is a member of $X$, i.e., $x_{1}-x_{2}$ is $m$-repeated low-density burst of length $b$ or less with weight $w$ or less, which is a contradiction.

Thus all the vectors in $X$ must belong to distinct cosets of the standard array. The number of such vectors over $\operatorname{GF}(q)$ is clearly $q^{m w}$. Also, total number of cosets in an $(n, k)$ linear code equals $q^{n-k}$, so we must have $q^{n-k} \geq q^{m w}$, i.e., $n-k \geq m w$, which proves the result.

Remark 4. For $m=2$, this result coincides with Theorem 1 of this paper for 2 -repeated low-density bursts of length $b$ or less with weight $w$ or less. For $m=2$ and $w=b$, this result coincides with the case of 2-repeated bursts of length $b$ or less (refer Theorem 1, Berardi, Dass and Verma [2]).

For $w=b$, this result reduces to the case when bursts considered are $m$ repeated bursts of length $b$ or less (refer Theorem 1, Dass and Verma [6]). For $m=1$ and $w=b$, this result reduces to the bound for the detection of bursts (refer Theorem 4.13, Peterson and Weldon [9]).

In the following, we consider linear codes which are capable to detect and correct simultaneously $m$-repeated low-density bursts with weight $w$ or less and obtain a necessary condition over the number of parity-checks required for such a code.

Theorem 5. Any $(n, k)$ linear code over $\mathrm{GF}(q)$ that corrects all $m$-repeated low-density bursts of length $b$ or less with weight $w$ or less $(w \leq b)$ must have at least $2 \mathrm{mw}$ parity-check digits. Further, if the code corrects all m-repeated low-density bursts of length $b$ or less with weight $w_{1}$ or less $\left(w_{1} \leq b\right)$ and simultaneously detects m-repeated low-density bursts of length $d$ or less $(d \geq b)$ with weight $w_{2}$ or less $\left(w_{2} \leq d\right)$, then the code must have at least $m\left(w_{1}+w_{2}\right)$ parity-check digits.

Proof. Consider a vector all of whose non-zero components are confined to the first $2 m b$ components. From each set of $b$ consecutive components, i.e., $(i b+1)$ th to $(i+1) b$-th components, $i=0,1, \ldots(2 m-1)$, the non-zero components are confined to some fixed $w(w \leq b)$ components. Such a vector is expressible as a sum or difference of two vectors each of which is $m$-repeated low-density burst of length $b$ or less with weight $w$ or less. These component vectors must belong to different cosets of the standard array because both such errors are correctable errors. Accordingly, such a vector viz., a burst of length $2 m b$ or 
less with weight $2 m w$ or less cannot be a code word. In view of Theorem 1 , such a code must have at least $2 m w$ parity-check digits.

Further, consider a vector whose only non-zero components are confined to the first $m(b+d)$ components. It may be noted that such a vector is expressible as a sum of $2 m$ vectors where $m$ of these are bursts of length $b$ or less each and the other $m$ are bursts of length $d$ or less each. From each set of $b$ consecutive components, the non-zero components are confined to some fixed $w_{1}\left(w_{1} \leq b\right)$ components whereas in each set of $d$ consecutive components, the non-zero components are confined to some fixed $w_{2}\left(w_{2} \leq d\right)$ components. Obviously, such a vector is expressible as a sum or difference of two vectors, one of which is $m$-repeated low-density burst of length $b$ or less with weight $w_{1}$ or less and the other is $m$-repeated low-density burst of length $d$ or less with weight $w_{2}$ or less. Both such component vectors, one being a detectable error and the other being a correctable error, cannot belong to the same coset of the standard array. Therefore, such a vector cannot be a code word, i.e., a low-density burst of length $m(b+d)$ with weight $m\left(w_{1}+w_{2}\right)$ or less cannot be a code vector. In view of Theorem 1 , such a code must have at least $m\left(w_{1}+w_{2}\right)$ parity-check digits.

Remark 5. For $m=2$, this result reduces to Theorem 3 of this paper for 2 -repeated low-density bursts of length $b$ or less with weight $w$ or less. For $m=2, w=w_{1}=b$ and $w_{2}=d$, the result coincides with the case of 2-repeated bursts of length $b$ or less (refer Theorem 3, Berardi, Dass, and Verma [2]).

For $w=w_{1}=b$ and $w_{2}=d$, this result reduces to Theorem 2 due to Dass and Verma [6] when bursts considered are $m$-repeated bursts of length $b$ or less. For $m=1, w=w_{1}=b$ and $w_{2}=d$, the result coincides with Reiger's bound (Reiger [10], also refer Theorem 4.15, Peterson and Weldon [9]).

We conclude the paper with an example of a 2-repeated low-density burst error detecting code of length 3 or less with weight 2 or less.

Example. Consider a $(9,4)$ binary code with parity-check matrix

$$
H=\left[\begin{array}{lllllllll}
1 & 0 & 0 & 0 & 0 & 0 & 1 & 1 & 0 \\
0 & 1 & 0 & 0 & 0 & 1 & 0 & 1 & 0 \\
0 & 0 & 1 & 0 & 0 & 1 & 0 & 0 & 1 \\
0 & 0 & 0 & 1 & 0 & 1 & 1 & 0 & 1 \\
0 & 0 & 0 & 0 & 1 & 1 & 0 & 1 & 1
\end{array}\right] .
$$

The matrix has been constructed by the synthesis procedure outlined in the proof of Theorem 2 by taking $b=3$ and $w=2$. It can be seen from Table 1 that the syndromes of different 2-repeated low-density bursts of length 3 or less with weight 2 or less are non-zero, showing thereby that the code that is the null space of this matrix detects all 2-repeated low-density bursts of length 3 or less with weight 2 or less. 
Table 1

\section{Error Vector-Syndrome}

\begin{tabular}{|c|c|}
\hline Error Vectors & Syndromes \\
\hline 100000000000 & 10000 \\
\hline 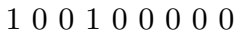 & 10010 \\
\hline $\begin{array}{lllllllll}1 & 0 & 0 & 0 & 1 & 0 & 0 & 0 & 0\end{array}$ & 100001 \\
\hline $\begin{array}{llllllllll}1 & 0 & 0 & 0 & 0 & 1 & 0 & 0 & 0\end{array}$ & 111111 \\
\hline 1000000100 & 000010 \\
\hline 10000000010 & $\begin{array}{lllll}0 & 10 & 0 & 1\end{array}$ \\
\hline $\begin{array}{lllllllll}1 & 0 & 0 & 0 & 0 & 0 & 0 & 0 & 1\end{array}$ & 10111 \\
\hline 1000111000000 & 10011 \\
\hline $\begin{array}{llllllllll}1 & 0 & 0 & 0 & 1 & 1 & 0 & 0 & 0\end{array}$ & 11110 \\
\hline 10000011100 & 011101 \\
\hline 1000000110 & 11011 \\
\hline $\begin{array}{lllllllll}1 & 0 & 0 & 0 & 0 & 0 & 0 & 1 & 1\end{array}$ & 01110 \\
\hline 100010010000 & 11101 \\
\hline 100001001000 & $\begin{array}{llllll}0 & 0 & 0 & 1 & 1\end{array}$ \\
\hline $\begin{array}{llllllllll}1 & 0 & 0 & 0 & 0 & 1 & 0 & 1 & 0\end{array}$ & 001110 \\
\hline $\begin{array}{lllllllll}10 & 0 & 0 & 0 & 0 & 1 & 0 & 1\end{array}$ & $\begin{array}{lllll}0 & 0 & 1 & 0 & 1\end{array}$ \\
\hline 11000000000 & 11000 \\
\hline 1110110000000 & 11010 \\
\hline $\begin{array}{lllllllll}1 & 1 & 0 & 0 & 1 & 0 & 0 & 0 & 0\end{array}$ & 11001 \\
\hline 11000010000 & 10111 \\
\hline 111000001000 & 01010 \\
\hline 11100000010 & $\begin{array}{llllll}0 & 0 & 0 & 0 & 1\end{array}$ \\
\hline $\begin{array}{lllllllll}1 & 1 & 0 & 0 & 0 & 0 & 0 & 0 & 1\end{array}$ & 11111 \\
\hline 110101100000 & 11011 \\
\hline 1110001110000 & 10110 \\
\hline 1110000111000 & $\begin{array}{llllll}0 & 0 & 1 & 0 & 1\end{array}$ \\
\hline $\begin{array}{llllllllll}1 & 1 & 0 & 0 & 0 & 0 & 1 & 1 & 0\end{array}$ & $\begin{array}{lllll}10 & 0 & 1 & 1\end{array}$ \\
\hline $\begin{array}{lllllllll}1 & 1 & 0 & 0 & 0 & 0 & 0 & 1 & 1\end{array}$ & 00110 \\
\hline 1110110100000 & 10101 \\
\hline 1110001001000 & $\begin{array}{llllll}0 & 1 & 0 & 1 & 1\end{array}$ \\
\hline 11000010010 & 01110 \\
\hline $\begin{array}{llllllllll}0 & 1 & 1 & 0 & 1 & 0 & 0 & 0 & 0\end{array}$ & 011101 \\
\hline $\begin{array}{llllllllll}0 & 1 & 1 & 0 & 0 & 1 & 0 & 0 & 0\end{array}$ & $\begin{array}{llllll}0 & 0 & 0 & 1 & 1\end{array}$ \\
\hline $\begin{array}{llllllllll}0 & 1 & 1 & 0 & 0 & 0 & 1 & 0 & 0\end{array}$ & 11110 \\
\hline $\begin{array}{llllllllll}0 & 1 & 1 & 0 & 0 & 0 & 0 & 1 & 0\end{array}$ & 10101 \\
\hline
\end{tabular}

\begin{tabular}{|c|c|}
\hline Error Vectors & Syndromes \\
\hline $\begin{array}{lllllllll}1 & 1 & 0 & 0 & 0 & 0 & 1 & 0 & 1\end{array}$ & $\begin{array}{llllll}0 & 1 & 1 & 0 & 1\end{array}$ \\
\hline 1010000000 & 10100 \\
\hline 10111000000 & 10110 \\
\hline 1010100000 & 10101 \\
\hline 10100010000 & 11011 \\
\hline 1010000100 & 00110 \\
\hline 1010000010 & 01101 \\
\hline 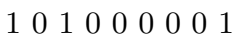 & 10011 \\
\hline 10111100000 & 10111 \\
\hline 101011000 & 11010 \\
\hline 10100011100 & $\begin{array}{llllll}0 & 1 & 0 & 0 & 1\end{array}$ \\
\hline 101000110 & 11111 \\
\hline 10100000011 & 01010 \\
\hline 10111010000 & 11001 \\
\hline 101010100 & 00111 \\
\hline 101001010 & 00010 \\
\hline 1010000101 & 000001 \\
\hline 01000000000 & 01000 \\
\hline 0100100000 & 01001 \\
\hline 01000010000 & 00111 \\
\hline $\begin{array}{lllllllll}0 & 1 & 0 & 0 & 0 & 0 & 1 & 0 & 0\end{array}$ & 11010 \\
\hline 01000000010 & 10001 \\
\hline $\begin{array}{lllllllll}0 & 1 & 0 & 0 & 0 & 0 & 0 & 0 & 1\end{array}$ & 011111 \\
\hline 010001110000 & 00110 \\
\hline $\begin{array}{lllllllll}0 & 1 & 0 & 0 & 0 & 1 & 1 & 0 & 0\end{array}$ & 10101 \\
\hline $\begin{array}{lllllllll}0 & 1 & 0 & 0 & 0 & 0 & 1 & 1 & 0\end{array}$ & $\begin{array}{lllll}0 & 0 & 0 & 1 & 1\end{array}$ \\
\hline 01000000011 & 10110 \\
\hline 01000101000 & 11011 \\
\hline 0100001010 & 11110 \\
\hline $\begin{array}{lllllllll}0 & 1 & 0 & 0 & 0 & 0 & 1 & 0 & 1\end{array}$ & 11101 \\
\hline 011100000000 & 01100 \\
\hline $\begin{array}{lllllllll}0 & 0 & 1 & 0 & 0 & 1 & 0 & 10\end{array}$ & 10010 \\
\hline $\begin{array}{lllllllll}0 & 0 & 1 & 0 & 0 & 0 & 1 & 0 & 1\end{array}$ & 10001 \\
\hline 000111000000 & 00110 \\
\hline 0001110010000 & 01001 \\
\hline
\end{tabular}




\begin{tabular}{|c|c|}
\hline Error Vectors & Syndromes \\
\hline $\begin{array}{llllllllll}0 & 1 & 1 & 0 & 0 & 0 & 0 & 0 & 1\end{array}$ & $0 \begin{array}{lllll}0 & 1 & 0 & 1 & 1\end{array}$ \\
\hline 011011000 & 00010 \\
\hline 011100011100 & 100001 \\
\hline 011100001110 & 000111 \\
\hline 011100000011 & 10010 \\
\hline 0111010100 & 11111 \\
\hline 0111001010 & 11010 \\
\hline 01110000101 & 11001 \\
\hline 0101000000 & 01010 \\
\hline $\begin{array}{lllllllll}0 & 1 & 0 & 1 & 1 & 0 & 0 & 0 & 0\end{array}$ & 01011 \\
\hline $\begin{array}{lllllllll}0 & 1 & 0 & 1 & 0 & 1 & 0 & 0 & 0\end{array}$ & 00101 \\
\hline 0101000100 & 11000 \\
\hline 010100010 & 10011 \\
\hline $\begin{array}{lllllllll}0 & 1 & 0 & 1 & 0 & 0 & 0 & 0 & 1\end{array}$ & 011101 \\
\hline $\begin{array}{lllllllll}0 & 1 & 0 & 1 & 1 & 1 & 0 & 0 & 0\end{array}$ & 00100 \\
\hline $\begin{array}{llllllllll}0 & 1 & 0 & 1 & 0 & 1 & 1 & 0 & 0\end{array}$ & 10111 \\
\hline 0101000110 & $\begin{array}{llllll}0 & 0 & 0 & 0 & 1\end{array}$ \\
\hline $\begin{array}{lllllllll}0 & 1 & 0 & 1 & 0 & 0 & 0 & 1 & 1\end{array}$ & 10100 \\
\hline 0101110100 & 110001 \\
\hline 010101010 & 11100 \\
\hline $\begin{array}{lllllllll}0 & 1 & 0 & 1 & 0 & 0 & 1 & 0 & 1\end{array}$ & 11101 \\
\hline $\begin{array}{llllllllll}0 & 0 & 1 & 0 & 0 & 0 & 0 & 0 & 0\end{array}$ & 00100 \\
\hline $\begin{array}{llllllllll}0 & 0 & 1 & 0 & 0 & 1 & 0 & 0 & 0\end{array}$ & 01011 \\
\hline 00100001000 & 10110 \\
\hline $\begin{array}{lllllllll}0 & 0 & 1 & 0 & 0 & 0 & 0 & 1 & 0\end{array}$ & 11101 \\
\hline $\begin{array}{lllllllll}0 & 0 & 1 & 0 & 0 & 0 & 0 & 0 & 1\end{array}$ & 000011 \\
\hline 001001100 & 11001 \\
\hline $\begin{array}{lllllllll}0 & 0 & 1 & 0 & 0 & 0 & 1 & 1 & 0\end{array}$ & 011111 \\
\hline $\begin{array}{lllllllll}0 & 0 & 1 & 0 & 0 & 0 & 0 & 1 & 1\end{array}$ & 11010 \\
\hline $\begin{array}{llllllllll}0 & 0 & 0 & 1 & 1 & 0 & 1 & 1 & 0\end{array}$ & 01000 \\
\hline 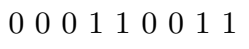 & 11101 \\
\hline $\begin{array}{lllllllll}0 & 0 & 0 & 1 & 1 & 0 & 1 & 0 & 1\end{array}$ & 10110 \\
\hline $\begin{array}{llllllllll}0 & 0 & 0 & 1 & 0 & 1 & 0 & 0 & 0\end{array}$ & 01101 \\
\hline $\begin{array}{llllllllll}0 & 0 & 0 & 1 & 0 & 1 & 1 & 0 & 0\end{array}$ & 11111 \\
\hline $\begin{array}{llllllllll}0 & 0 & 0 & 1 & 0 & 1 & 0 & 1 & 0\end{array}$ & 10100 \\
\hline $\begin{array}{lllllllll}0 & 0 & 0 & 1 & 0 & 1 & 0 & 0 & 1\end{array}$ & 01010 \\
\hline $\begin{array}{llllllllll}0 & 0 & 0 & 1 & 0 & 1 & 1 & 1 & 0\end{array}$ & 00110 \\
\hline $\begin{array}{lllllllll}0 & 0 & 0 & 1 & 0 & 1 & 0 & 1 & 1\end{array}$ & 10011 \\
\hline
\end{tabular}

\begin{tabular}{|c|c|}
\hline Error Vectors & Syndromes \\
\hline 00111000100 & 10100 \\
\hline $\begin{array}{llllllllll}0 & 0 & 1 & 1 & 0 & 0 & 0 & 1 & 0\end{array}$ & 11111 \\
\hline $\begin{array}{lllllllll}0 & 0 & 1 & 1 & 0 & 0 & 0 & 0 & 1\end{array}$ & $\begin{array}{llllll}0 & 0 & 0 & 0 & 1\end{array}$ \\
\hline $\begin{array}{llllllllll}0 & 0 & 1 & 1 & 0 & 1 & 1 & 0 & 0\end{array}$ & $\begin{array}{lllll}1 & 1 & 0 & 1 & 1\end{array}$ \\
\hline $\begin{array}{llllllllll}0 & 0 & 1 & 1 & 0 & 0 & 1 & 1 & 0\end{array}$ & $\begin{array}{llllll}0 & 1 & 1 & 0 & 1\end{array}$ \\
\hline $\begin{array}{lllllllll}0 & 0 & 1 & 1 & 0 & 0 & 0 & 1 & 1\end{array}$ & 11000 \\
\hline $\begin{array}{lllllllll}0 & 0 & 1 & 1 & 0 & 1 & 0 & 1 & 0\end{array}$ & 10000 \\
\hline $\begin{array}{lllllllll}0 & 0 & 1 & 1 & 0 & 0 & 1 & 0 & 1\end{array}$ & 10011 \\
\hline $\begin{array}{lllllllll}0 & 0 & 1 & 0 & 1 & 0 & 0 & 0 & 0\end{array}$ & $\begin{array}{lllll}0 & 0 & 1 & 0 & 1\end{array}$ \\
\hline $\begin{array}{llllllllll}0 & 0 & 1 & 0 & 1 & 1 & 0 & 0 & 0\end{array}$ & 01010 \\
\hline $\begin{array}{llllllllll}0 & 0 & 1 & 0 & 1 & 0 & 1 & 0 & 0\end{array}$ & 10111 \\
\hline $\begin{array}{lllllll}0 & 0 & 1 & 0 & 1 & 0 & 0\end{array}$ & 11100 \\
\hline $\begin{array}{lllllllll}0 & 0 & 1 & 0 & 1 & 0 & 0 & 0 & 1\end{array}$ & 00010 \\
\hline $\begin{array}{lllllllll}0 & 0 & 1 & 0 & 1 & 1 & 0 & 0\end{array}$ & 11000 \\
\hline $\begin{array}{lllllllll}0 & 0 & 1 & 0 & 1 & 0 & 1 & 1 & 0\end{array}$ & 011110 \\
\hline $\begin{array}{lllllllll}0 & 0 & 1 & 0 & 1 & 0 & 0 & 1 & 1\end{array}$ & 11011 \\
\hline 000100111010 & 10011 \\
\hline $\begin{array}{llllllllll}0 & 0 & 1 & 0 & 1 & 0 & 1 & 0 & 1\end{array}$ & 10000 \\
\hline $\begin{array}{llllllllll}0 & 0 & 0 & 1 & 0 & 0 & 0 & 0 & 0\end{array}$ & 00010 \\
\hline $\begin{array}{llllllllll}0 & 0 & 0 & 1 & 0 & 0 & 1 & 0 & 0\end{array}$ & 10000 \\
\hline $\begin{array}{llllllllll}0 & 0 & 0 & 1 & 0 & 0 & 0 & 1 & 0\end{array}$ & 11011 \\
\hline $\begin{array}{lllllllll}0 & 0 & 0 & 1 & 0 & 0 & 0 & 0 & 1\end{array}$ & $\begin{array}{llllll}0 & 0 & 1 & 0 & 1\end{array}$ \\
\hline $\begin{array}{lllllllll}0 & 0 & 0 & 1 & 0 & 0 & 1 & 1 & 0\end{array}$ & 010 \\
\hline $\begin{array}{lllllllll}0 & 0 & 0 & 1 & 0 & 0 & 0 & 1 & 1\end{array}$ & 111 \\
\hline $\begin{array}{llllllllll}0 & 0 & 0 & 1 & 0 & 0 & 1 & 0 & 1\end{array}$ & 10111 \\
\hline $\begin{array}{llllllllll}0 & 0 & 0 & 1 & 1 & 0 & 0 & 0 & 0\end{array}$ & $\begin{array}{llllll}0 & 0 & 0 & 1 & 1\end{array}$ \\
\hline $\begin{array}{llllllllll}0 & 0 & 0 & 1 & 1 & 0 & 1 & 0 & 0\end{array}$ & 10001 \\
\hline 00001110010 & 11010 \\
\hline 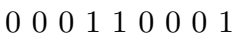 & $\begin{array}{llllll}0 & 0 & 1 & 0 & 0\end{array}$ \\
\hline $\begin{array}{llllllllll}0 & 0 & 0 & 0 & 0 & 1 & 0 & 1 & 0\end{array}$ & 10110 \\
\hline $\begin{array}{llllllllll}0 & 0 & 0 & 0 & 0 & 1 & 0 & 1 & 1\end{array}$ & $\begin{array}{lllll}1 & 0 & 0 & 0 & 1\end{array}$ \\
\hline $\begin{array}{llllllllll}0 & 0 & 0 & 0 & 0 & 0 & 1 & 0 & 0\end{array}$ & 10010 \\
\hline $\begin{array}{llllllllll}0 & 0 & 0 & 0 & 0 & 0 & 1 & 1 & 0\end{array}$ & $\begin{array}{lllll}0 & 1 & 0 & 1 & 1\end{array}$ \\
\hline $\begin{array}{lllllllll}0 & 0 & 0 & 0 & 0 & 0 & 1 & 0 & 1\end{array}$ & 10101 \\
\hline 1111110000000 & 11110 \\
\hline 1110100000 & 11101 \\
\hline $\begin{array}{llllllllll}0 & 1 & 1 & 1 & 1 & 0 & 0 & 0 & 0\end{array}$ & 01111 \\
\hline $\begin{array}{lllllllll}0 & 1 & 1 & 1 & 0 & 1 & 0 & 0 & 0\end{array}$ & $\begin{array}{lllll}0 & 0 & 0 & 0 & 1\end{array}$ \\
\hline
\end{tabular}

Contd. 


\begin{tabular}{|c|c|}
\hline Error Vectors & Syndromes \\
\hline $\begin{array}{lllllllll}0 & 0 & 0 & 1 & 0 & 1 & 1 & 0 & 1\end{array}$ & 11000 \\
\hline $\begin{array}{lllllllll}0 & 0 & 0 & 0 & 1 & 0 & 0 & 0 & 0\end{array}$ & $\begin{array}{llllll}0 & 0 & 0 & 0 & 1\end{array}$ \\
\hline $\begin{array}{lllllllll}0 & 0 & 0 & 0 & 1 & 0 & 0 & 1 & 0\end{array}$ & 11000 \\
\hline $\begin{array}{lllllllll}0 & 0 & 0 & 0 & 1 & 0 & 0 & 0 & 1\end{array}$ & $\begin{array}{llllll}0 & 0 & 1 & 1 & 0\end{array}$ \\
\hline $\begin{array}{llllllllll}0 & 0 & 0 & 0 & 1 & 0 & 0 & 1 & 1\end{array}$ & 111111 \\
\hline 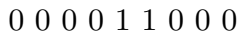 & 011110 \\
\hline $\begin{array}{lllllllll}0 & 0 & 0 & 0 & 1 & 1 & 0 & 1 & 0\end{array}$ & $\begin{array}{lllll}10 & 1 & 1 & 1\end{array}$ \\
\hline $\begin{array}{lllllllll}0 & 0 & 0 & 0 & 1 & 1 & 0 & 0 & 1\end{array}$ & $\begin{array}{llll}0 & 10 & 0 & 1\end{array}$ \\
\hline $\begin{array}{llllllllll}0 & 0 & 0 & 0 & 1 & 1 & 0 & 1 & 1\end{array}$ & 10000 \\
\hline $\begin{array}{llllllllll}0 & 0 & 0 & 0 & 1 & 0 & 1 & 0 & 0\end{array}$ & 10011 \\
\hline 0000010110 & 01010 \\
\hline $\begin{array}{lllllllll}0 & 0 & 0 & 0 & 1 & 0 & 1 & 0 & 1\end{array}$ & 10100 \\
\hline $\begin{array}{llllllllll}0 & 0 & 0 & 0 & 1 & 0 & 1 & 1 & 1\end{array}$ & $\begin{array}{lllll}0 & 1 & 1 & 0 & 1\end{array}$ \\
\hline $\begin{array}{llllllllll}0 & 0 & 0 & 0 & 0 & 1 & 0 & 0 & 0\end{array}$ & 011111 \\
\hline $\begin{array}{llllllllll}0 & 0 & 0 & 0 & 0 & 1 & 0 & 0 & 1\end{array}$ & 01000 \\
\hline $\begin{array}{llllllllll}0 & 0 & 0 & 0 & 0 & 1 & 1 & 0 & 0\end{array}$ & 11101 \\
\hline $\begin{array}{lllllllll}0 & 0 & 0 & 0 & 0 & 1 & 1 & 0 & 1\end{array}$ & 11010 \\
\hline
\end{tabular}

\begin{tabular}{|c|c|}
\hline Error Vectors & Syndromes \\
\hline $\begin{array}{llllllllll}0 & 0 & 1 & 1 & 1 & 1 & 0 & 0 & 0\end{array}$ & 010000 \\
\hline $\begin{array}{lllllllll}0 & 0 & 1 & 1 & 1 & 0 & 1 & 0 & 0\end{array}$ & $\begin{array}{lllll}10 & 1 & 0 & 1\end{array}$ \\
\hline $\begin{array}{lllllllll}0 & 0 & 0 & 1 & 1 & 1 & 1 & 0 & 0\end{array}$ & 11110 \\
\hline $\begin{array}{llllllllll}0 & 0 & 0 & 1 & 1 & 1 & 0 & 1 & 0\end{array}$ & 1010101 \\
\hline $\begin{array}{llllllllll}0 & 0 & 0 & 0 & 1 & 1 & 1 & 1 & 0\end{array}$ & $\begin{array}{llllll}0 & 0 & 1 & 0 & 1\end{array}$ \\
\hline $\begin{array}{lllllllll}0 & 0 & 0 & 0 & 1 & 1 & 1 & 0 & 1\end{array}$ & 11011 \\
\hline $\begin{array}{lllllllll}0 & 0 & 0 & 0 & 0 & 1 & 1 & 1 & 1\end{array}$ & $\begin{array}{llllll}0 & 0 & 0 & 1 & 1\end{array}$ \\
\hline 111100000000 & 11100 \\
\hline $\begin{array}{llllllllll}0 & 1 & 1 & 1 & 0 & 0 & 0 & 0 & 0\end{array}$ & $\begin{array}{lllll}0 & 1 & 1 & 1 & 0\end{array}$ \\
\hline $\begin{array}{llllllllll}0 & 0 & 1 & 1 & 1 & 0 & 0 & 0 & 0\end{array}$ & 001111 \\
\hline 000011110000 & 01100 \\
\hline $\begin{array}{llllllllll}0 & 0 & 0 & 0 & 1 & 1 & 1 & 0 & 0\end{array}$ & 11100 \\
\hline $\begin{array}{llllllllll}0 & 0 & 0 & 0 & 0 & 1 & 1 & 1 & 0\end{array}$ & $\begin{array}{llllll}0 & 0 & 1 & 0 & 0\end{array}$ \\
\hline $\begin{array}{llllllllll}0 & 0 & 0 & 0 & 0 & 0 & 1 & 1 & 1\end{array}$ & 011100 \\
\hline $\begin{array}{llllllllll}0 & 0 & 0 & 0 & 0 & 0 & 0 & 1 & 0\end{array}$ & 11001 \\
\hline $\begin{array}{lllllllll}0 & 0 & 0 & 0 & 0 & 0 & 0 & 1 & 1\end{array}$ & 11110 \\
\hline $\begin{array}{lllllllll}0 & 0 & 0 & 0 & 0 & 0 & 0 & 0 & 1\end{array}$ & $\begin{array}{llllll}0 & 0 & 1 & 1 & 1\end{array}$ \\
\hline
\end{tabular}

\section{References}

[1] N. M. Abramson, A class of systematic codes for non-independent errors, IRE Trans. IT-5 (1959), 150-157.

[2] L. Berardi, B. K. Dass, and R. Verma, On 2-repeated burst error detecting codes, Journal of Statistical Theory and Practice 3 (2009), no. 2, 381-391.

[3] B. K. Dass, Bounds for codes detecting burst-errors with weight constraints, Proc. Conf. on Measures of Information and Their Applications, Indian Institute of Technology, Bombay, India, August 16-18, 1974.

[4] __ A sufficient bound for codes correcting bursts with weight constraint, J. Assoc. Comput. Mach. 22 (1975), no. 4, 501-503.

[5] B. K. Dass and R. Verma, Repeated burst error correcting linear codes, Asian-Eur. J. Math. 1 (2008), no. 3, 303-335.

[6] _ Repeated burst error detecting linear codes, Ratio Mathematica-Journal of Applied Mathematics 19 (2009), 25-30.

[7] P. Fire, A Class of Multiple-Error-Correcting Binary Codes for Non-Independent Errors, Sylvania Reports RSL-E-2, Sylvania Reconnaissance Systems, Mountain View, California, 1959.

[8] R. W. Hamming, Error detecting and error correcting codes, Bell System Tech. J. 29 (1950), 147-160.

[9] W. W. Peterson and E. J. Weldon, Jr., Error-Correcting Codes, Second edition. The M.I.T. Press, Cambridge, Mass.-London, 1972.

[10] S. H. Reiger, Codes for the correction of "clustered" errors, IRE Trans. IT-6 (1960), $16-21$.

[11] G. E. Sacks, Multiple error correction by means of parity checks, IRE Trans. IT-4 (1958), 145-147.

[12] B. D. Sharma and B. K. Dass, Extended Varshamov-Gilbert and sphere-packing bounds for burst-correcting codes, IEEE Trans. Information Theory IT-20 (1974), 291-292. 
[13] A. D. Wyner, Low-density burst-error-correcting codes, IEEE Trans. Information Theory IT-9 (1963), no. 2, p. 124.

B. K. DASS

Department of Mathematics

UNIVERSITY OF DELHI

DELHI - 110 007, INDIA

E-mail address: dassbk@rediffmail.com

RASHMI VERMA

Department of Mathematics

UNIVERSITY OF DELHI

DELHI - 110 007, INDIA

E-mail address: riva_7@rediffmail.com 